\title{
Design and demonstration of a distributed-coupling linear accelerator structure
}

\author{
Sami Tantawi, ${ }^{1}$ Mamdouh Nasr, ${ }^{1, *}$ Zenghai Li,${ }^{1}$ Cecile Limborg, ${ }^{1}$ and Philipp Borchard ${ }^{2}$ \\ ${ }^{1}$ SLAC National Accelerator Laboratory, 94025 California, USA \\ ${ }^{2}$ Dymenso LLC, San Francisco, 94124 California, USA
}

(Received 3 June 2019; revised 8 July 2020; accepted 20 July 2020; published 10 September 2020)

\begin{abstract}
We present a topology for linear accelerators (linacs) in which the power is distributed to the cavities through a waveguide with periodic apertures that guarantees the correct phases and amplitudes along the structure length. Unlike conventional traveling and standing-wave linacs, the presented topology allows the cavity shapes to be designed without the constraints applied to the coupling between cells to transfer power from one cell to the next. Therefore, the topology permits more degrees of freedom for the optimization of individual cavity shapes in comparison with conventional linacs. The cavity shapes can be optimized for power consumption and efficiency, and/or the manipulation of the surface fields for high gradient operation. This topology also provides a possibility for low-temperature manufacturing techniques that prevent the annealing of the material during typical brazing processes; hence, the material could retain its original properties such as hardness. We present a design and an experimental demonstration of this linac.
\end{abstract}

DOI: 10.1103/PhysRevAccelBeams.23.092001

\section{INTRODUCTION}

A linear particle accelerator (linac) accelerates charged particles using an oscillating electric field formed within rf cavities (cells) that are joined together to form a beam line. Charged particles gain energy as they travel along the beam line. Typically for high gradient electron linacs, such as the SLAC linear accelerator, rf power is fed to the linac from one point and flows through adjacent cells using coupling holes that also serve as a beam tunnel for charged particles. Consequently, the linac design process requires careful consideration of the coupling between adjacent cells. This limits the ability of designers to optimize the cell shape for efficiency and gradient handling capability [1-3]. Biperiodic structures and side coupled structures have been introduced $[4,5]$ to avoid coupling between cells through the beam tunnel and hence allow for the ability of better optimization of the cavity shapes. These topologies enjoy widespread use in industrial and medical applications. However, they have not been considered for high gradient linacs aimed at discovery science machines such as future linear colliders and linac-based free electron lasers.

Standing-wave linacs, based on biperiodic and side coupled-cavity linac topologies, use an extra set of cavities,

\footnotetext{
Corresponding author. mamdouh@slac.stanford.edu

Published by the American Physical Society under the terms of the Creative Commons Attribution 4.0 International license. Further distribution of this work must maintain attribution to the author(s) and the published article's title, journal citation, and DOI.
}

the coupling cavities, either on the side or on axis, to achieve the correct phase between accelerating cavities. These coupling cavities do not contribute to the acceleration, and hence, they are made very short in case of the onaxis coupling or moved to the side in case of the side coupled-cavity topology. The coupling cavities are supposed to be located at the null of a standing wave pattern of these standing wave accelerator structures. Still, power must flow from the feeding point, typically close to the middle of the linac, to the cells at the far end of the linac. The power indeed flows from one cell to the next, including the coupling cavities, and the coupling slots have to accommodate this power flow; the power for the whole linac has to flow through the coupler cavity that couples the feeding waveguide to the linac. Hence the side cavities do contribute to the total $\mathrm{rf}$ losses in the linac, and the power flowing through the coupling slots of the coupler cavity is the power needed to feed the whole linac.

The distributed coupling linac, presented here, has a topology that allows feeding each accelerator cell independently using a periodic feeding network. This adds another degree of freedom to the design; the coupling between cells can have a wide acceptable range, including minimizing it to negligible values. The added flexibility in the geometry optimization of the accelerator cells can be used to attain the highest possible shunt impedance for a set of cells or craft the field along the cell walls to allow for higher gradient operation [6].

In Sec. II we introduce the concept, in Sec. III we detail the design procedure for the accelerating cell and the coupling slots, and in Sec. IV we present the mechanical 
design, then, in Sec. V, we present the experimental verification of our manufactured linac structure. Finally, Sec. VI introduces variations of the distributed-coupling linac and compare it with other topologies.

\section{DISTRIBUTED COUPLING LINAC THEORY: THE DISTRIBUTION MANIFOLD}

Any concept for a distributed feeding network is required to provide, typically, an equal amount of power to each identical cell and simultaneously provide the appropriate phased advance for each cell. For a typical electron linac with particle moving at nearly the speed of light, the phase advance per cell is $2 \pi P / \lambda$; where $P$ is the periodic separation between cells and $\lambda$ is the free-space wavelength. Except for a dielectric-free coaxial line, this phase advance cannot be provided by any form of waveguiding structure which always has a guided wavelength $\lambda_{g}>\lambda$, the freespace wavelength.

Using a coaxial line to feed a set of cavities has been attempted before $[7,8]$. These approaches have not been used since these initial publications; the use of a coaxial line is difficult for many reasons. Supporting the inner conductor without dielectric support throughout the linac length poses manufacturing difficulties. The mode transducer that would couple the input waveguide to the linac to the coaxial line requires finesse in design to ensure low field levels and will have to be incorporated in the center conductor support structure. Furthermore, in a reasonable design, the coaxial line needs to support only the TEM mode. The cutoff frequency of the next higher-order mode, the $\mathrm{TE}_{11}$ mode, limits the size of the coaxial line. For the lowest possible losses, it is readily shown that the ratio between the inner and outer conductor radii should be $\sim 0.28[9,10]$. Using this ratio and demanding that the $\mathrm{TE}_{11}$ cutoff frequency is reasonably above the operating frequency requires that the outer conductor radius be $\sim 0.26 \lambda$, where $\lambda$ is the operating wavelength. With these limitations on the coaxial line dimensions, the losses per meter at $11.424 \mathrm{GHz}$, our operating frequency of interest, is about $5 \%$. Also, the peak field at the inner conductor is about $45 \mathrm{MV} / \mathrm{m}$ for an input power of $100 \mathrm{MW}$, a typical number for the operation of advanced high gradient linacs [11,12]. These numbers are to be compared with $2 \%$ of losses/meter and $28 \mathrm{MV} / \mathrm{m}$ of peak field if a reasonably sized waveguide is used. Hence, it was natural for us to consider an approach to the distribution system that uses easy to manufacture structure with an integrated waveguide distribution system.

Our approach uses waveguides that are oriented so that their center E-plane coincides with an E-plane of the accelerator cell's $\mathrm{TM}_{010}$ mode, which is any plane that contains the center axis of the accelerator cell. Because the guided wavelength does not match the free-space wavelength, one can imagine solutions where the distribution waveguide is bent like a serpentine to achieve the

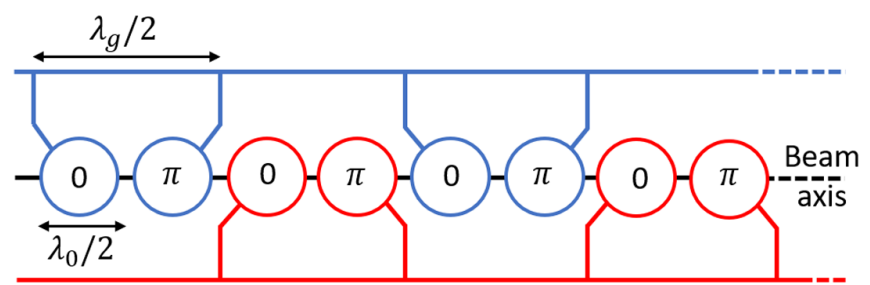

FIG. 1. The distributed feeding network for $\pi$-mode linac with two manifolds feeding interleaving pairs of cavities; every two adjacent cavities require a $\pi$ phase shift, which is provided by successive tap-offs from the manifold, separated by $\lambda_{g} / 2$.

appropriate phase advance. This is valid, but one can use more than a single distribution manifold (by a manifold we mean a waveguide branching into several openings to feed many cavities). A natural interval for taping into the manifold, as would be seen below, is every $\left(m \lambda_{g}\right) / 2$; where $\lambda_{g}$ is the guided wavelength within the manifold, and $m$ is an integer. Therefore, for a structure with a phase advance/ cavity of $2 \pi / n$, one can use $n$ manifolds, each one of them being taped every $\lambda_{g} / 2$ with an interleaving $\pi$ phase delay incorporated within the cell feed lines; then, every cell is fed by a given manifold have the same phase. Since we have total freedom of choosing the phase advance between cells, the phase advance can be viewed as an optimization parameter.

A $\pi$ phase advance per cavity is a special case that simplifies the design of the system. In this case, $n=2$, and hence, only two manifolds are needed. It turns out that this period is close to being optimal. For small beam apertures, a phase advance of $2 \pi / 3$ is few percents better than the $\pi$ phase advance in terms of shunt impedance ([13] and Sec. III of this paper). However, for simplicity, we developed our initial design, presented here, around a linac operating at the $\pi$-mode. The manifold, in this case, can also be simplified by supplying the power from the manifold to one cavity every $\lambda_{g} / 2$. Transverse fields of the fundamental mode of a waveguide switch polarity every $\lambda_{g} / 2$. Since every two adjacent cavities require a $\pi$ phase shift, then two successive tap-offs from the manifold, separated by $\lambda_{g} / 2$ can feed those two adjacent cavities. Hence, the two manifolds would feed interleaving pairs of cavities (see Fig. 1). In this case, there is no need to modify the feeding line for every other cell as suggested before; feeding lines are the same for all the cavities.

For this topology, the manifold's network consists of a set of cascaded T-junctions. The power going through the tap-off ports to the cavities should achieve a minimal standing-wave ratio (SWR) within the feeding lines to the cavities; the feeding network, then, will have minimal influence on the cavity cells, resulting in two isolated systems, the feeding network and the cavity cells. To this end, the cavity coupling port should be designed to be a matched port when the cavity is loaded with the design 


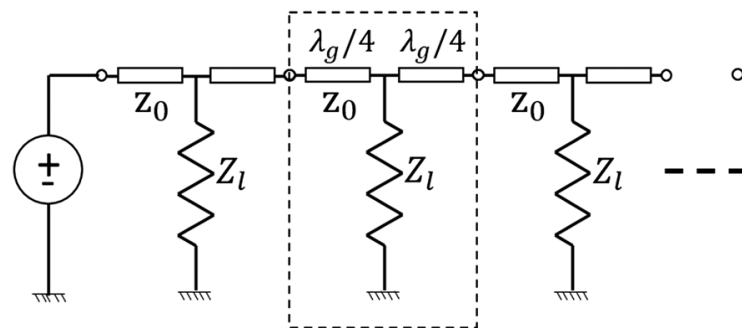

(a)

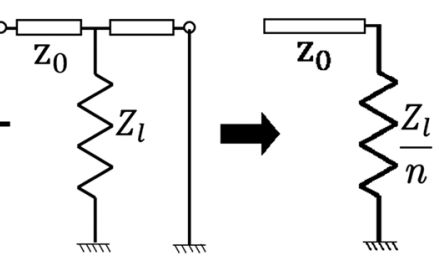

(b)

FIG. 2. (a) The isolated manifold system can be viewed as a transmission line with periodic loading with identical loads that has an impedance $Z_{l}$ located at $i \lambda g / 2$; where $i \in \mathbb{Z}$ (b) the total load impedance can be shown with the help of elementary transmission line theory to be $Z_{l} / n$.

beam current. For the $\pi$-mode, the accelerator's cells can be regarded as isolated from each other with no coupling in between. This is true only for the $\pi$-mode and is independent of the size of the coupling hole size; see Sec. VI, the simulation section, for more details. Therefore, the cells can be modeled as independent loads using a general model (resonant circuit) for the cavity input impedance. The isolated manifold system, with the assumption of a matched load at the end of each tap-off port, can be viewed as a transmission line with periodic loading and a short circuit termination at the end, situated $\lambda_{g} / 4$ away from the last load, see Fig. 2(a). Because all the loads are identical, the total load impedance, seen by the input source, can be shown, with the help of elementary transmission line theory to be $Z_{l} / n$, see Fig. 2(b). Hence, for a matched input, we choose $Z_{l}=n Z_{0}$, where $Z_{0}$ is the characteristic impedance of the line. With this choice, the line is segmented into a series of sections with a load impedance of value $n Z_{0}$ in the middle of the section and a quarter-wave transmission line extending the section from either direction. This section can be viewed as a two-port network. Using scattering matrix representation which could be immediately derived from the impedance matrix representation for each section [14], we can write the scattering matrix, after using the appropriate signs for the field polarities, for each section as

$$
S_{\mathrm{two}}=\left(\begin{array}{cc}
\frac{-1}{2 n+1} & \frac{1}{2 n+1}-1 \\
\frac{1}{2 n+1}-1 & \frac{-1}{2 n+1}
\end{array}\right) \text {, }
$$

The scattering matrix representation, $S$, of the lossless three-port tap-off section can then be deduced. The $2 \times 2$ matrix entries of Eq. (1) have to be the same as the upper left submatrix of $S$. Also, $S$ has to be symmetrical because of reciprocity. Finally, because it is lossless, it satisfies the unitarity condition; $S S^{\dagger}=I$. The geometric symmetry of the system is expressed in this matrix if $s_{11}=s_{22}$ and $s_{13}=-s_{23}$; the negative sign that differentiates $s_{13}$ from $s_{23}$ expresses the $\pi$ phase shift across each section. Hence,

$$
S=\left(\begin{array}{ccc}
\frac{-1}{2 n+1} & \frac{1}{2 n+1}-1 & \frac{2 i \sqrt{n}}{2 n+1} \\
\frac{1}{2 n+1}-1 & \frac{-1}{2 n+1} & \frac{-2 i \sqrt{n}}{2 n+1} \\
\frac{2 i \sqrt{n}}{2 n+1} & \frac{-2 i \sqrt{n}}{2 n+1} & \frac{2 n-1}{2 n+1}
\end{array}\right)
$$

The next stage of the implantation requires the translation of this matrix into a realizable physical structure. This is done with the aid of a more general scattering matrix representation of any three-port junction satisfying unitarity, reciprocity, and geometrical symmetry, but without satisfying the particular form of Eq. (2). This matrix is derived in [15] and is given by

$S=\left(\begin{array}{ccc}\frac{-e^{i \phi}-\cos \theta}{2} e^{i 2 \psi} & \frac{-e^{i \phi}+\cos \theta}{2} e^{i 2 \psi} & \frac{\sin \theta}{\sqrt{2}} e^{i \vartheta} e^{i \psi} \\ \frac{-e^{i \phi}+\cos \theta}{2} e^{i 2 \psi} & \frac{-e^{i \phi}-\cos \theta}{2} e^{i 2 \psi} & \frac{-\sin \theta}{\sqrt{2}} e^{i \vartheta} e^{i \psi} \\ \frac{\sin \theta}{\sqrt{2}} e^{i \vartheta} e^{i \psi} & \frac{-\sin \theta}{\sqrt{2}} e^{i \vartheta} e^{i \psi} & \cos \theta e^{i 2 \vartheta}\end{array}\right)$.

After imposing reciprocity, unitarity and geometrical symmetry conditions, there are only 4 degrees of freedoms left, represented by the angles $\theta, \varphi, \psi$, and $\vartheta$. Equation (3) indicates that in general, we need 4 degrees of freedom in our geometrical implementation for a junction that satisfies Eq (2). The physical structure is shown in Fig. 3(a) for the T-junction, with features labeled $\mathbf{0}$ and $\mathbf{2}$, having fourdimensional degrees of freedom in terms of the depth and width of each feature. The first feature, which is a capacitive element at the feeding port, provides control to achieve the design value for $\left|s_{33}\right|$ or $\theta$ and $\vartheta$, while the second one controls $\varphi$, and $\psi$. Performing a numerical search through a finite element code quickly determines the correct dimensions for this junction so that it satisfies Eq. (2).

After designing one single junction, the manifold can be constructed by cascading these junctions $n$ times. We numerically simulated a manifold designed for $n=10$ at X-band. The results are shown in Fig. 3(b). The coupling between the input port and any tap-off port is $-10 \mathrm{~dB}$ and the phase difference between any two adjacent tap-offs is $\pi$. 

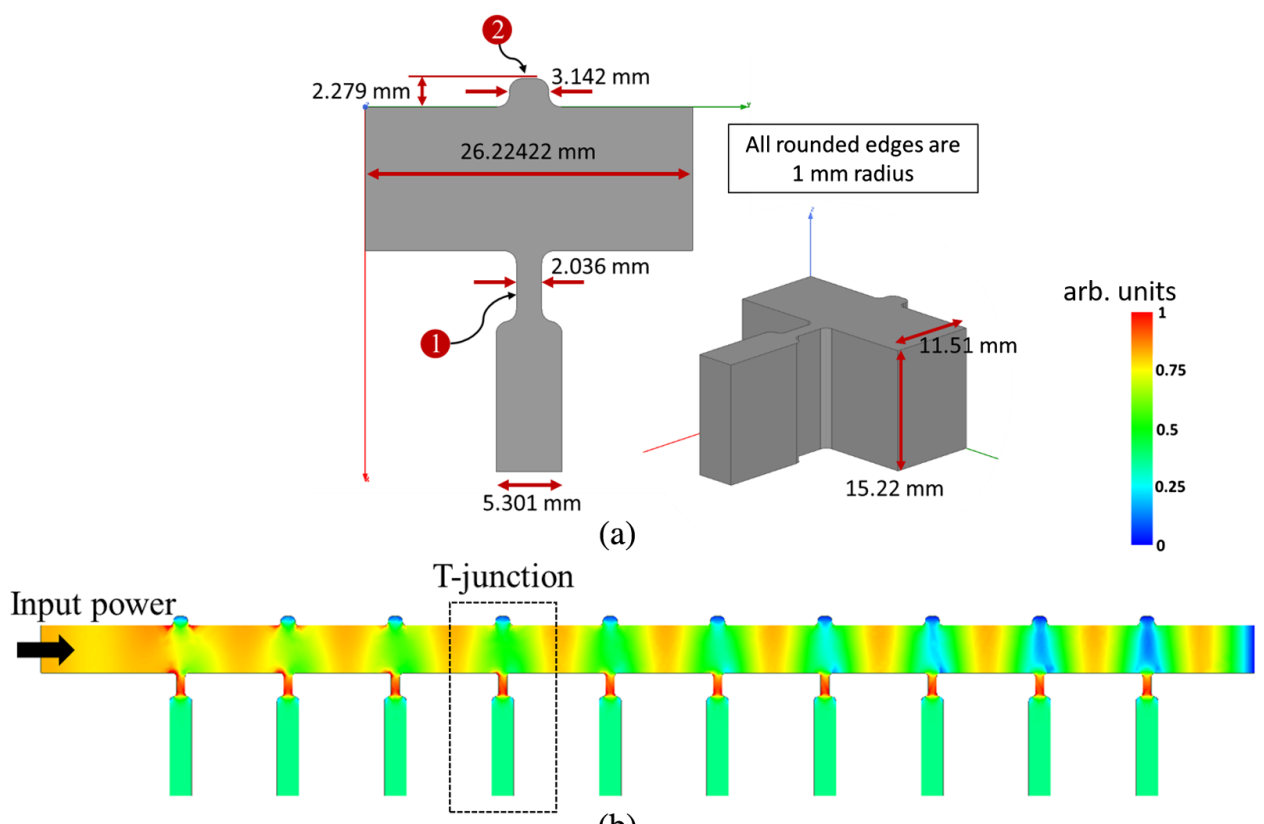

(b)

FIG. 3. (a) The T-junction, with features labeled $\mathbf{1}$ and $\mathbf{2}$, have four-dimensional degrees of freedom in terms of the depth and width of each feature. Performing a numerical search through a finite element code quickly determines the correct dimensions for this junction so that it satisfies Eq. (2) for $n=10$ in this example, then (b) the full manifold is constructed by cascading this junction 10 times. The color plot is for the complex E-field simulated using ANSYS HFSS.

As described, this practical implementation of a distributed feeding network provides independent power feeding to each accelerator cell, and thus enables designing for completely isolated cells. In this case, the accelerator design converges to a single-cell design with identical accelerator cells. This simplicity of the design concept opens the door to investigate new accelerator cell geometries.

\section{CELL OPTIMIZATION AND NUMERICAL SIMULATION OF THE DISTRIBUTED COUPLING LINAC}

For our first design, presented here, we used a set of parameters defining a series of circular and straight segments to describe the geometry of the accelerator cell. These parameters, in Fig. 4(a), are optimized using
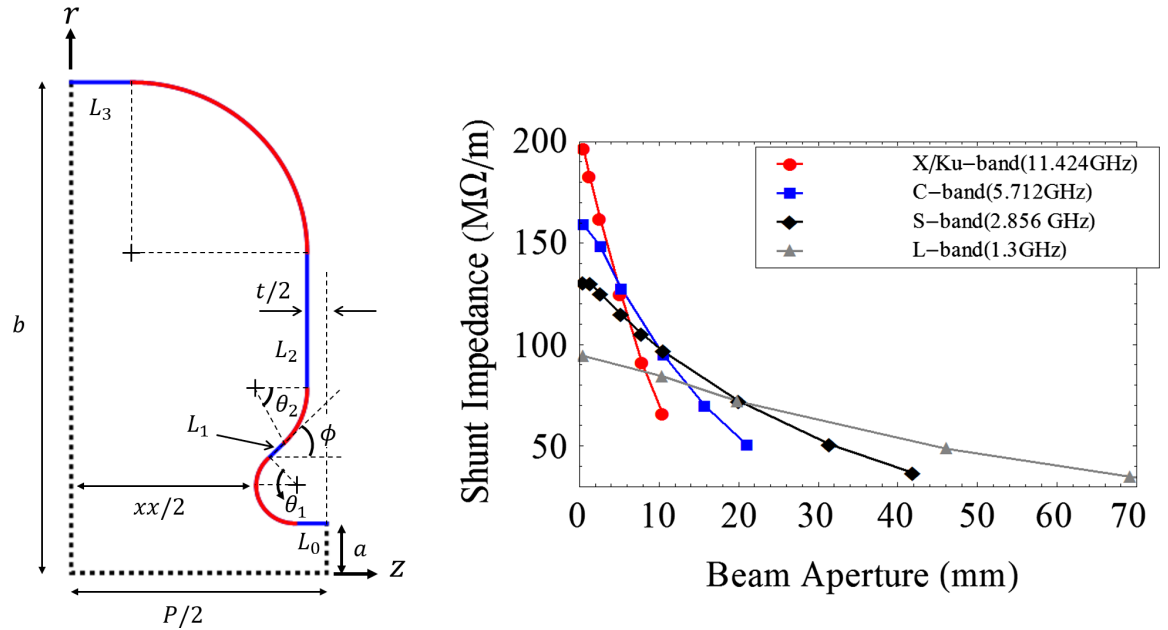

FIG. 4. (a) The accelerating cell is defined using a series of circular and straight segments. The radii of the cavity segments are then optimized to maximize/minimize the optimization function that is defined by the user. (b) The shunt impedance for optimized designs utilizing the distributed-coupling technology for different aperture openings at different bands. 


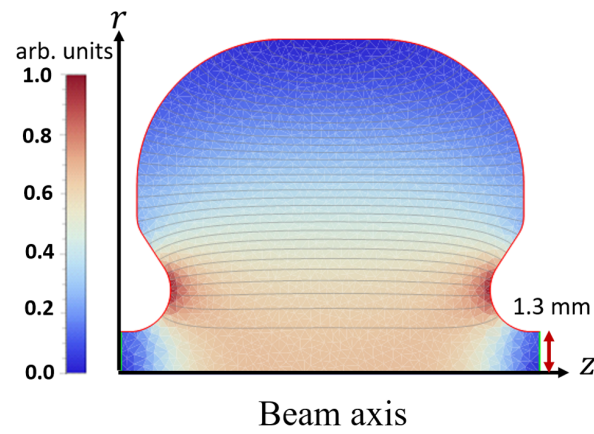

(a)

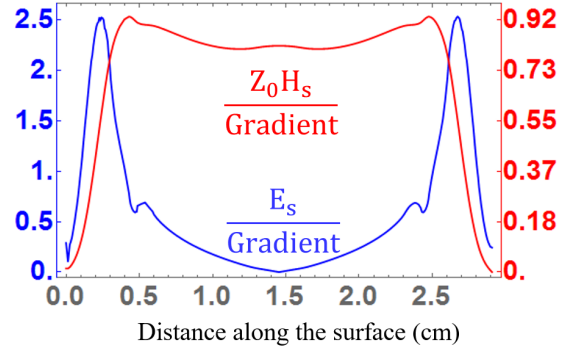

(b)

FIG. 5. (a) The optimized cell design and electric field profile plot. (b) A plot of the surface electric and magnetic fields for the optimized cell design for X-band linac with iris diameter of $2.6 \mathrm{~mm}$.

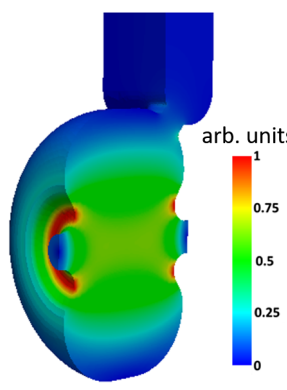

(a)

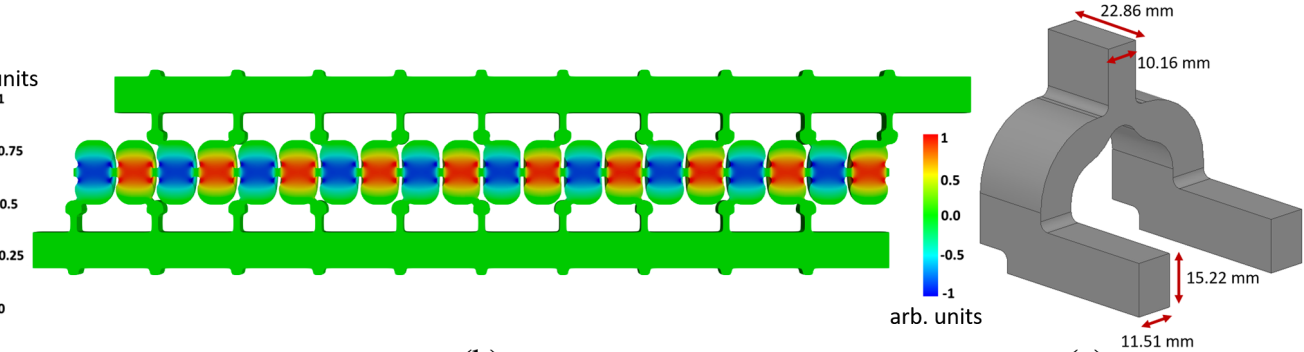

(c)

FIG. 6. (a) The cell-design shape with the feeding port and electric field profile plot. (b) The full design and electric field profile of 20 cells $\pi$-mode distributed-coupling linac with identical cells at X-band. (c) The power divider designed to distribute the power equally between the two feeding manifolds from a WR90 feeding port.

differential evolution to maximize the shunt impedance with a constraint on the peak fields on the cavity surface. We used our well-developed 2D finite element (FEM) codes in our optimizations. Typically, an optimization problem takes a few hours using our 2D FEM codes compared to a few days using commercially available software. Our optimizations, in Fig. 4(b), show that the shunt impedance improves for smaller aperture openings for all bands. Consequently, designing for isolated cells with the distributed coupling concept enables reaching high duty factors compared to any conventional particle accelerator with high power handling capability.

The first realization of the distributed-coupling linac is designed for $\pi$-mode operation at X-band (11.424 GHz) with an iris diameter of $2.6 \mathrm{~mm}$. The cell geometry was optimized for minimal peak magnetic field on the surface; following many studies that showed the high correlation between breakdown rates in accelerator structures and the peak magnetic fields on the accelerator walls [16-19]. The optimized design, in Fig. 5(a), achieves a superior shunt impedance of $155 \mathrm{M} \Omega / \mathrm{m}$. The minimized magnetic field on the surface came at the cost of a peak electric field-togradient ratio of 2.5 as shown in Fig. 5(b). Meanwhile, the shunt impedance of linac structures which are designed for high-gradient $\mathrm{X}$-band operation is in the order of $100 \mathrm{M} \Omega / \mathrm{m}$ with a comparable peak electric field-togradient ratio $[12,20]$.

The distributed feeding network is designed for 20-cells linac; ten cells per manifold. As previously mentioned, the S-matrix in Eq. (2) for $n=10$ was achieved by introducing two features, labeled $\mathbf{0}$ and 2 in Fig. 3(a), to the T-junction. The feeding ports, in Fig 6(a), are designed for critical coupling for each accelerator cell, and the full linac design is shown in Fig. 6(b). We also designed a power divider that

TABLE I. Summary of the cavity accelerating design parameters. The peak fields are for an average gradient of $100 \mathrm{MV} / \mathrm{m}$.

\begin{tabular}{lc}
\hline \hline Frequency & $11.424 \mathrm{GHz}$ \\
Q0 & 10000 \\
Qext & 10000 \\
Length & $10 \lambda(\sim 26 \mathrm{~cm})$ \\
Iris diameter & $2.6 \mathrm{~mm}$ \\
Shunt impedance & $155 \mathrm{M} \Omega / \mathrm{m}$ \\
Peak surface E & $250 \mathrm{MV} / \mathrm{m}$ \\
Peak surface H & $0.25 \mathrm{MA} / \mathrm{m}$ \\
Peak surface H with the feeding port & $0.575 \mathrm{MA} / \mathrm{m}$ \\
$S_{c}[16]$ & $7.36 \mathrm{~W} / \mu \mathrm{m}^{2}$ \\
\hline \hline
\end{tabular}




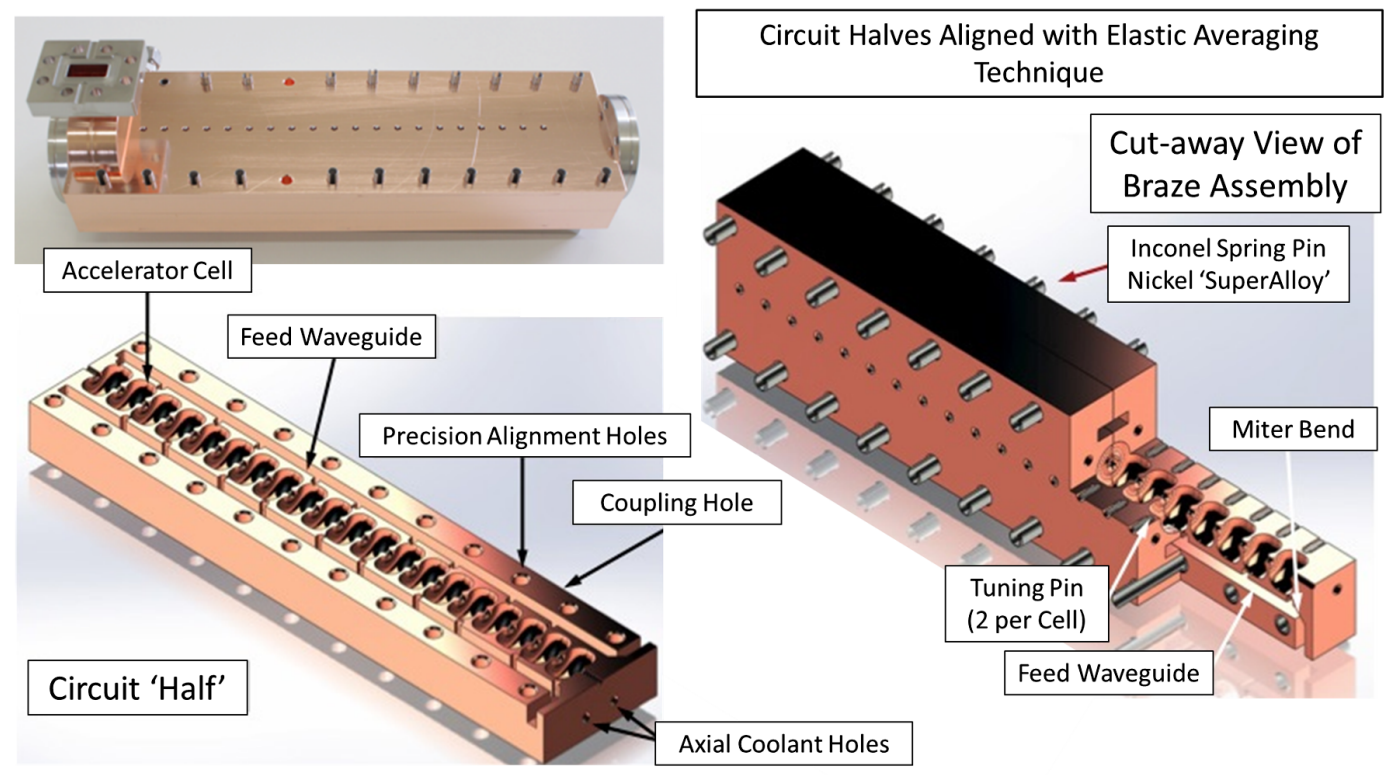

FIG. 7. Illustration of the manufacturing of the distributed-coupling linac from two quasi-identical copper blocks including the tuning pins, alignment holes, and cooling channels.

divides the power equally between the two feeding manifolds with minimal reflections. The power divider has WR90 feeding port and splits into two branches that match the dimensions of the manifolds as shown in Fig. 6(c).

A summary of the linac parameters is presented in Table I. We must mention, however, that for the design presented in this paper, we did not optimize the feeding ports to the accelerator cells for minimal enhancement of the magnetic field. This led to a magnetic field enhancement of $x 2.3$ on the surface. In our later designs, we optimized the feeding ports to reduce the enhancement factor to only x1.1-1.2, depending on the beam-loading requirements [21]. We also developed advanced optimization approaches that utilize elliptical and even generic shapes for more optimized accelerator cells [22].

\section{MECHANICAL DESIGN OF THE STRUCTURE}

Each segment of the distributed-coupling accelerator structure can be manufactured from two blocks as shown in Fig. $7[6,23,24]$. Both the manifolds and the cavities have no currents crossing the plane which splits the structure in half along the long dimension of the manifold cross section. Manufacturing from two blocks reduces the complexity of manufacturing the structure and provides logical places for both the cooling tubes and the tuning holes. It also provides a possibility for low-temperature manufacturing techniques including electron beam welding and thereby allowing the use of unannealed copper alloys and hence greater tolerance to high gradient operation. The circuit halves are aligned with an elastic averaging technique $[25,26]$.

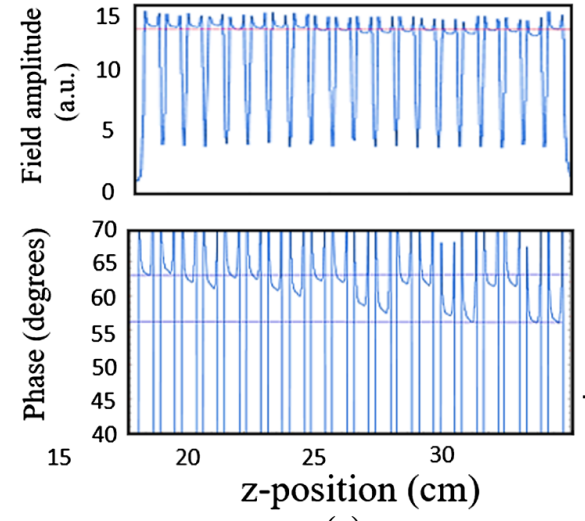

(a)

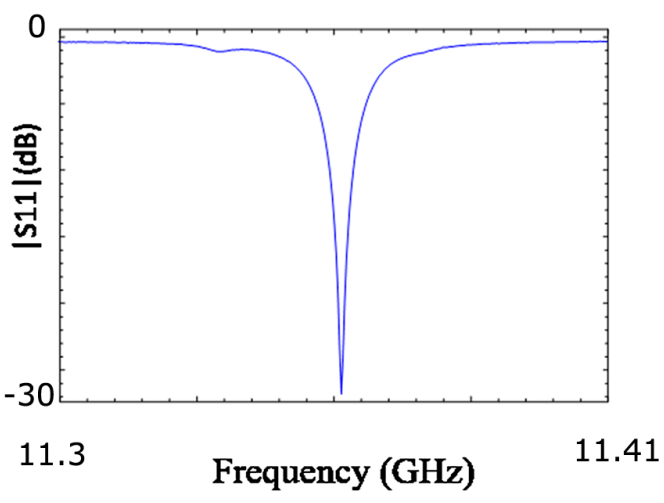

(b)

FIG. 8. The cold test data for the manufactured 20-cells X-band standing-wave distributed-coupling linac showing (a) the amplitude and phase along the structure as well as (b) the frequency spectrum. The structure features a single resonance frequency rather than 20 resonances for coupled linac structures. 


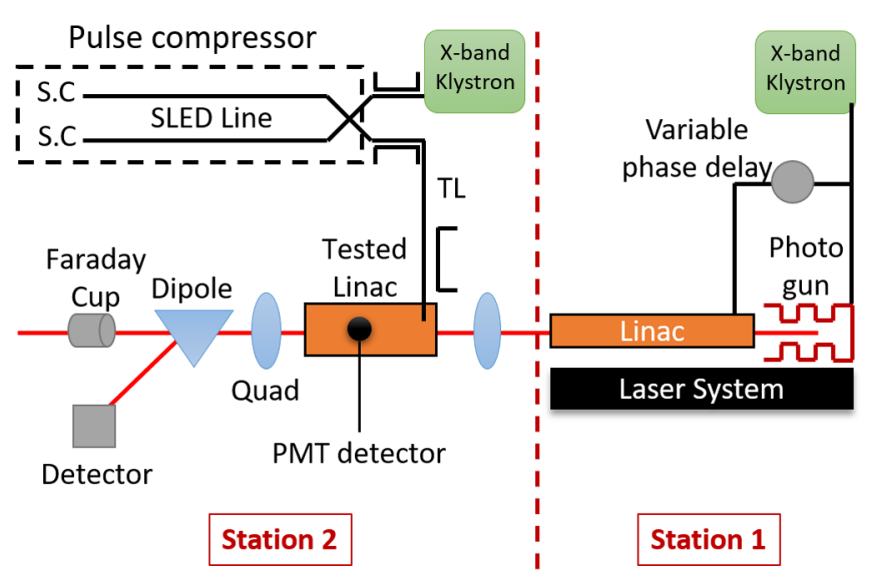

FIG. 9. The experimental setup for the distributed coupling linac testing at XTA, NLCTA, SLAC. XTA is divided into two stations, and can be referred to as station 1 and station 2 . The first station generates the electron beam used in the experiment, and the distributed-couping linac is installed in the second station.

The accelerator structure was manufactured and cold tested before high power operation. Tuning was accomplished by shorting all the cells, with a conductive rod, except the one cell being tuned. The resultant structure after tuning, as expected, had a single resonance frequency rather than 20 resonances for conventional structures with 20 coupled accelerator cells [1]. Figure 8 shows the frequency response data together with the bead pull [27] data that characterizes the amplitude and phase along the structure. It is worth mentioning that the side coupled linac cannot be manufactured from two blocks due to the geometry of the side coupling cells which also needs careful tuning for proper power flow.

\section{EXPERIMENTAL RESULTS}

We utilized the existing infrastructure at the X-band Test Accelerator (XTA) station at the NLCTA facility at SLAC for the high-power experiments of the distributed-coupling linac. The goal is to experimentally verify the linac parameters from Table I at high-power operation. The experimental setup is illustrated in Fig. 9. XTA is divided into two stations, referenced in Fig. 9 as stations 1 and 2; each one is fed with an independent rf source. The first station is used to generate the electron beam from an $\mathrm{X}$-band photoinjector and then accelerate the beam to about 63.5 MeV using an NLC style X-band accelerator [28]. The second station feeds the distributed-coupling linac, which is installed after the station 1 linac.

An X-band klystron feeds the distributed-coupling linac with $\mathrm{rf}$ power. The peak $\mathrm{rf}$ power of the klystron is $40 \mathrm{MW}$, and we installed a pulse compressor (multimoded SLED-II [29]) after the klystron to push the rf power above the klystron limit. We installed the pulse compressor after the distributedcoupling linac successfully demonstrated high-gradient operation and was limited by the available klystron power.

Quadrupoles are used for beam focusing and trajectory adjustment, a spectrometer is placed after the distributedcoupling linac to measure the beam energy, and a Faraday cup (FC) is installed downstream for charge measurements. Also, a photomultiplayer tube (PMT) is placed on top of the structure to measure the bremsstrahlung radiation from the linac and detect breakdowns in the copper surface.

The accelerating gradient of a linac is expressed, from the basic definition of the shunt impedance, $R_{s}$, and cavity filling equations as

$$
\begin{aligned}
\text { Gradient } & =\sqrt{\frac{R_{s} P_{\text {loss }}}{L}}\left(1-e^{-\frac{t}{t_{F}}}\right), \\
t_{F} & =\frac{2 Q_{0}}{(1+\beta) \omega_{0}} \quad \text { with } \quad \beta=Q_{0} / Q_{\text {ext }},
\end{aligned}
$$

where $P_{\text {loss }}$ is the steady-state value of the power loss in the structure, and $L$ is the linac length. Because the structure is

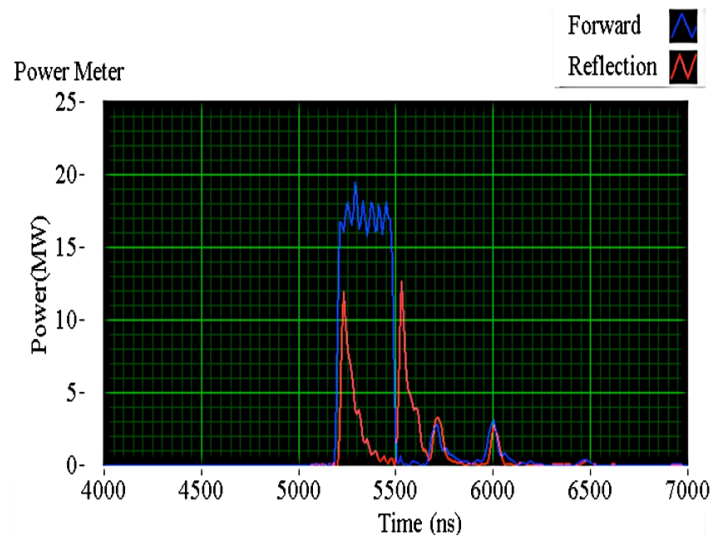

(a)

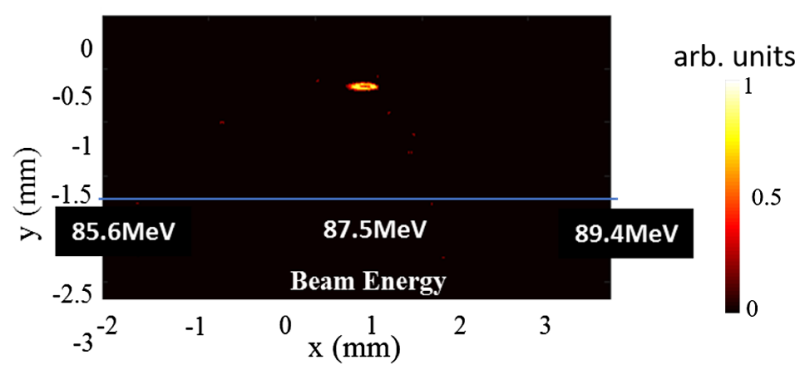

(b)

FIG. 10. The energy gain of a synchronized electron-beam entering the structure with an energy of $63.5 \mathrm{MeV}$. (a) The structure was fed with a square pulse of $300 \mathrm{~ns}$ and a power level of $17.5 \mathrm{MW}$ as shown. (b) The energy of the accelerated electron bunch is $87.5 \mathrm{MeV}$; i.e., energy gain is $24 \mathrm{MeV}$. 


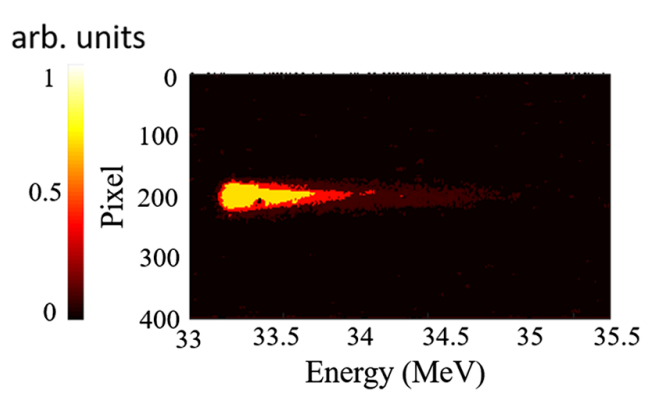

(a)

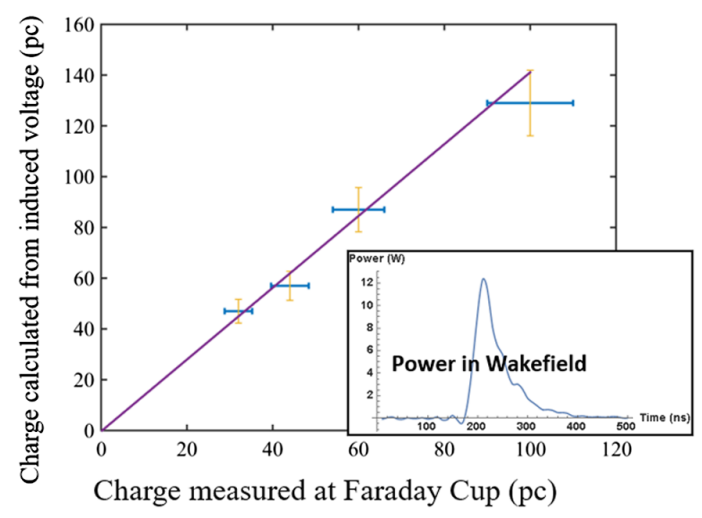

(b)

FIG. 11. (a) The peak dark-current energy at simulated gradient of $140 \mathrm{MV} / \mathrm{m}$, and (b) comparison of the measured charge from Faraday cup and calculated using the cavity parameters and the induced wakefield.

critically coupled $\left(Q_{0}=Q_{\text {ext }}\right.$ from Table I), then $P_{\text {loss }}=$ $P_{\text {in }}$ at steady state. Substituting with the parameters from Table I, the peak gradient for the distributed-coupling linac is expressed as

$$
\operatorname{Gradient}\left(\frac{\mathrm{MV}}{m}\right)=24.303 \sqrt{P_{\text {in }}(\mathrm{MW})}\left(1-e^{-\frac{t(n s)}{140}}\right)
$$

for a square pulse with a width of $t(n s)$. This equation will be used to simulate the structure gradient in our following measurements.

First, we measured the energy gain of an electron beam moving down the axis of the distributed-coupling linac and compared the result with our calculations from Eq. (5). A 300 ns square rf pulse at 17.5 MW, in Fig. 10(a), fed the linac with power. Substituting with the pulse parameters in Eq. (5), the simulated gradient is $89.74 \mathrm{MV} / \mathrm{m}$ which translates to energy gain of $23.3 \mathrm{MeV}$ for our $0.26 \mathrm{~m}$ linac. The electron beam had initial energy of $63.5 \mathrm{MeV}$ and exited the linac at $87.5 \mathrm{MeV}$ as measured by the spectrometer in Fig. 10(b). Thus, measuring an energy gain of $24 \mathrm{MeV}$, this is in large agreement with the simulated value using the cavity parameters.

Second, at high-gradient levels, charges migrate from the copper surface of the linac forming observable dark current. Some of these charges get captured and accelerated on axis; the peak dark-current energy corresponds to the peak energy gain of the linac. After installing the pulse compressor, with 400 ns stepped rf input pulse with a steady state power of $33 \mathrm{MW}$, the linac reached a high gradient of $140 \mathrm{MV} / \mathrm{m}$ corresponding to energy gain of $36.74 \mathrm{MeV}$ as calculated from Eq. (5); this is in large agreement with the measured peak dark-current energy of $35.7 \mathrm{MeV}$ in Fig. 11(a).

Third, a beam moving down the axis of the linac excites wakefields related to the total charge of the beam. We measured the wakefield power and then used the cavity parameters in Table I to calculate the total charge of the electron beam. The calculated charge is compared with the measured value using the FC downstream showing very good agreement in Fig. 11(b). The detailed calculation is presented in the Appendix.

\section{VARIATIONS OF DISTRIBUTED-COUPLING LINAC AND COMPARISONS WITH OTHER TOPOLOGIES}

The distributed-coupling scheme is especially advantageous for multicell structures with small on-axis coupling. In such structures, the frequency separation between the $\pi$-mode and the next $\frac{N-1}{N} \pi$-mode can be very small and not feasible to be driven with a conventional coupler due to potential coupling to the nearby modes. For example, the structure presented in Sec. III has a total bandwidth of $0.34 \mathrm{MHz}$ while the frequency separation between the $\pi$ and the $\frac{N-1}{N} \pi$ mode is merely $2 \mathrm{kHz}$ which is well within the bandwidth of the $\pi$-mode resonance. The typical coupling scheme would excite both the operating $\pi$-mode as well as the $\frac{N-1}{N} \pi$ mode (and a few more nearby modes). However, the mode coupling issue is eliminated with the parallel waveguide feeding scheme.

We should point out that the distributed-coupling topology is also applicable to any phase advance per cell and not necessarily $\pi$-mode. The only condition is for the iris diameter to be small enough to eliminate the coupling

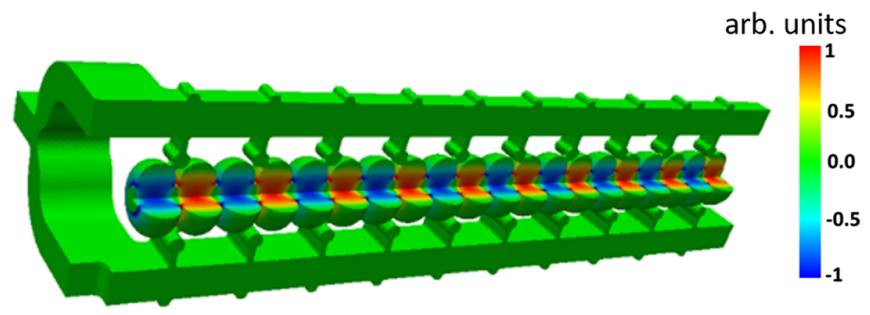

FIG. 12. Parallel feed of a 20-cell structure with large on-axis coupling. The contour plot is the Ez field, showing the $\pi$ phase advance. 


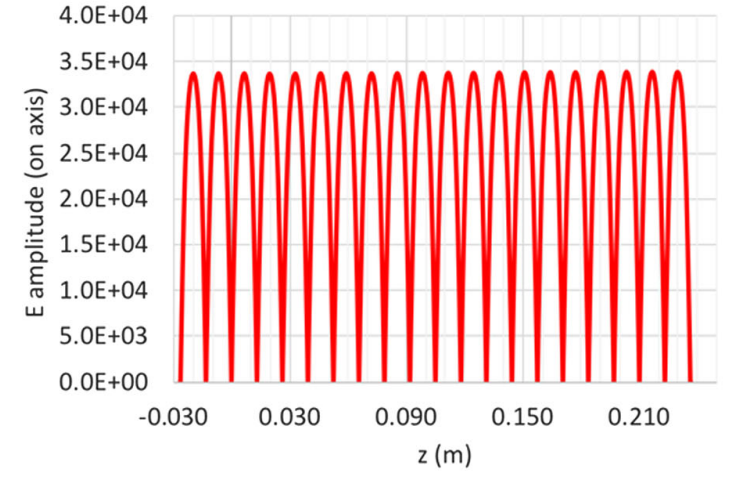

(a)

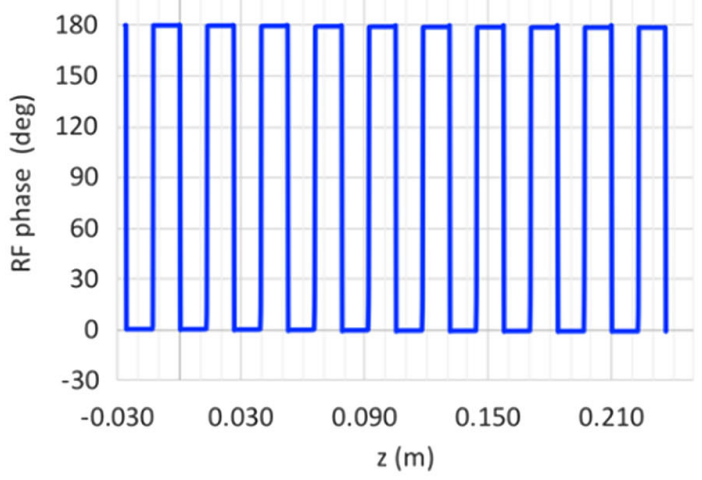

(b)

FIG. 13. (a) The electric field amplitude on axis, with arbitrary units; (b) the $\pi$-mode phase in the 20-cell structure.

between adjacent cells. Phase delays can then be incorporated within the feeding lines to each accelerating cell to achieve the required phase advance between cells. The $\pi$-mode is an exception where the parallel feeding scheme, however, is equally applicable to structures of significant cell-to-cell coupling. The reason is that the $\pi$-mode has a zero group velocity, thus naturally providing the needed isolation between the cells irrespective of the iris diameter.

Figure 12 shows a 20-cell structure with a large iris opening fed by the distributed-coupling system. The total bandwidth of this structure is $256 \mathrm{MHz}$, and the frequency spacing between the $\pi$ and the $\frac{N-1}{N} \pi$-mode is $2 \mathrm{MHz}$ which is far outside of the bandwidth of the $\pi$-mode resonance. The parallel feed waveguide naturally drives the $\pi$-mode of the structure, producing flat acceleration fields with an exact $180^{\circ}$ phase advance as shown in Fig. 13.

A conventional way of powering a weakly coupled multicell structure is by means of side coupling cavities

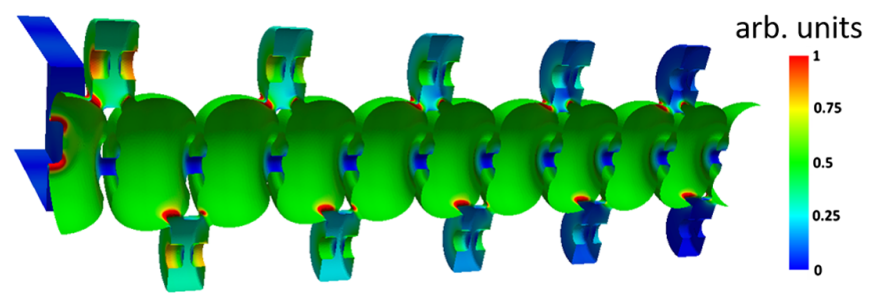

FIG. 14. Side coupled accelerator structure. Shown here is the downstream half of a 20-cell structure. The rectangular waveguide coupler is in the middle of the 20 cells.
[5]. With the side coupled cavities, the structure forms a $\pi / 2$ mode. In a lossless case, the $\pi / 2$ mode will have zero fields in the side coupled cavities. In reality, however, power will flow through the side coupled cavities, as shown in Fig. 14 to compensate for the cavity surface loss and beam loading if there is any, resulting in finite power loss in the side coupling cavities and a phase shift in the accelerating cavities.

The coupling slot between the side coupling cavity and the accelerating cavity will also induce a local field enhancement, which could be optimized with certain limitations; the red spots in Fig. 14 indicate the magnetic field enhancement around these coupling slots. The power loss on the side cavity walls of the side coupled structure is compared with that of the feeding waveguide of the parallel feeding scheme. Both structures are designed to have the critical coupling (with Q0 of room temperature copper). The accelerating cells are identical in both structures.

Figure 15 shows the surface magnetic contour of half of a 20-cell parallel feed structure. Both the power delivering structures (the side cavities in the side coupled structure, and the waveguide in the parallel feeding structure) power the same number of accelerator cells. The magnetic fields in Figs. 14 and 15 are normalized with the same scaling for comparison. One can see finite fields in the side coupling cavities due to power flow, while the fields in the parallel feed waveguide are of a much lower level. The surface loss in the side coupling cavities is $2.6 \%$ of the surface loss of the accelerating cavities in a side coupled structure. This ratio is $1.14 \%$ in the parallel feed structure between the

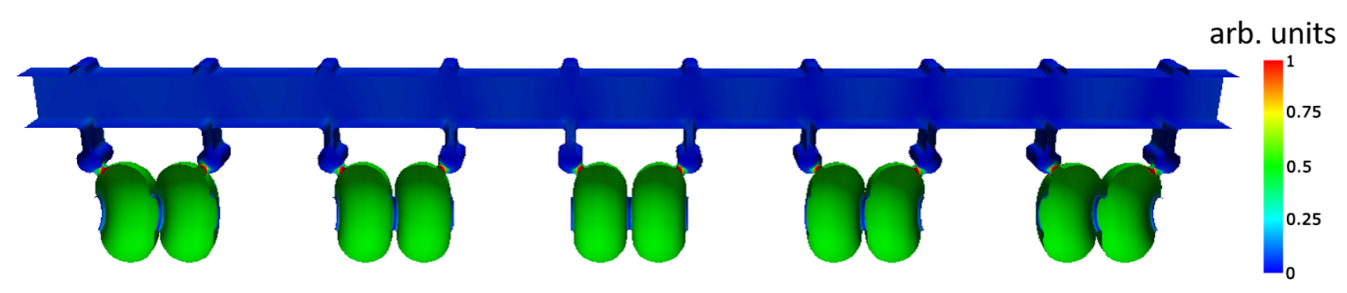

FIG. 15. The surface magnetic contour of one manifold for a 20-cell parallel feed structure. 
waveguide and the cavity losses, which is more than a factor of 2 lower.

\section{CONCLUSION}

In summary, we developed a new linac architecture that feeds each accelerator cell independently. The distributedcoupling linac overcomes the limitations imposed on the cell shape to satisfy cell-to-cell coupling conditions; it hence allows for flexible geometry optimization of the accelerator cells. Consequently, the resultant designs achieve minimized surface fields, enabling high gradient operation, and maximized shunt impedance, enabling efficient operation. We also developed a new manufacturing technique that uses only two copper blocks to build the new linac architecture, enabling economical manufacturing. We applied the distributed coupling linac concept to a 20-cells X-band design and achieved a superior shunt impedance of $155 \mathrm{M} \Omega / \mathrm{m}$. We tested the linac for high power operation and verified the designed shunt impedance using different experimental measurements. The distributed coupling linac topology opens the door for many applications and design spaces that include multifrequency operation $[30,31]$, and can be extended to superconducting accelerators $[21,32]$.

\section{ACKNOWLEDGMENTS}

This work is supported by the U.S. Department of Energy (DOE) under Contract No. DE-AC0276SF00515, DOE SLAC Laboratory-Directed Research and Development award, and Stanford School of Medicine. rf structure simulations were performed at the DOE's NERSC supercomputing facilities at Lawrence Berkeley National Laboratory. The authors would like to thank Prof. Billy W. Loo Jr. and Prof. Peter Maxim for the many useful discussions and the support provided by the Stanford Medical school.

\section{APPENDIX: CALCULATIONS OF BEAM CHARGE USING THE MEASUREMENT OF THE INDUCED WAKEFIELDS}

From the fundamental beam loading theory [33-35], the total voltage gain by an electron beam equals the voltage gain provided by the linac, $V_{a}$, minus the induced voltage from wakefields, $V_{s}$. From reciprocity, the total voltage gained by the beam when the linac is operating equals the induced voltage by the beam when the linac is off:

$$
V_{\text {total }}=V_{a}-V_{s}=V_{s} \rightarrow V_{a}=2 V_{s} .
$$

Substituting from Eq. (A1) into the definition of the shunt impedance,

$$
R_{s}=\frac{\text { Gradient }^{2}}{P_{\text {loss }} / L}=\frac{\left(V_{a} / L\right)^{2}}{P_{\text {loss }} / L}=\frac{4 V_{s}^{2}}{P_{\text {loss }} L} .
$$

Substituting for $V_{s}$ with the stored energy, $U$, and using the basic definition of the quality factor, we can express the beam charge as follows:

$$
R_{s}=\frac{4|U|^{2}}{q^{2} P_{\text {loss }} L}=\frac{4 Q_{0}|U|}{q^{2} \omega_{0} L} \rightarrow \therefore q^{2}=\frac{4 Q_{0}}{R_{s} \omega_{0} L}|U|
$$

From the conservation of energy,

$$
\frac{d U}{d t}=P_{\text {in }}-P_{\text {loss }}-P_{\text {ref }},
$$

where, in the described case, the linac is off, thus $P_{\text {in }}=0$, and the reflected power equals the emitted power, $P_{e}$, from wakefields:

$$
\therefore P_{\text {ref }}=P_{e}=\frac{\omega_{0}|U|}{Q_{e}}=\beta P_{\text {loss }} \text {. }
$$

Substituting from Eq. (A5) into Eq. (A4), we can express the stored energy as

$$
U=-\left(1+\beta^{-1}\right) \int P_{\text {ref }} d t .
$$

Substituting from Eq. (A6) into Eq. (A3), the total beam charge can be calculated from the measured reflected power as

$$
q=2(1+\beta) \sqrt{\frac{Q_{t}}{\beta R_{s} \omega_{0} L}} \sqrt{\int P_{\text {ref }} d t .}
$$

[1] C. Karzmark, C. Nunan, and E. Tanabe, Medical Electron Accelerators (McGraw-Hill, Incorporated, Health Professions Division, 1993), Chap. 3, ISBN: 9780071054102.

[2] T. Wangler, Rf Linear Accelerators (Wiley, New York, 2008). Chap. 4.

[3] T. Maury and C. Wu, Handbook Of Accelerator Physics And Engineering, 3rd printing (World Scientific Publishing Company, Singapore, 1999).

[4] E. A. Knapp, Linear Accelerator Conference, 1964 (1964) MURA-714, p. 31.

[5] E. A. Knapp, B. C. Knapp, and J. M. Potter, Standing wave high energy linear accelerator structures, Rev. Sci. Instrum. 39, 979 (1968).

[6] S. G. Tantawi, Z. Li, and P. Borchard, Distributed coupling and multifrequency microwave accelerators, U.S. Patent No. 9 386, 682 (2016). 
[7] R. Sundelin, J. Kirchgessner, and M. Tigner, Parallel coupled cavity structure, IEEE Trans. Nucl. Sci. 24, 1686 (1977).

[8] V.A. et al., in 11th All-Union Workshop on Accel. Of Charged Part. Dubna (1989), Vol. 1, p. 268.

[9] C. S. Franklin, British Patent No. 284, 005 (1928).

[10] E. I. Green, F. A. Leibe, and H. E. Curtis, The proportioning of shielded circuits for minimum high-frequency attenuation, Bell Syst. Tech. J. 15, 248 (1936).

[11] P. B. Wilson, Prospects for very high gradient linaccolliders, IEEE Trans. Nucl. Sci. 28, 2742 (1981).

[12] A. Grudiev and W. Wuensch, Design of the CLIC main linac accelerating structure for CLIC conceptual design report, Report No. EuCARD-CON-2010-073, 2010, https://accelconf.web.cern.ch/LINAC2010/papers/mop068 .pdf.

[13] R. H. Miller, Comparison of standing wave and traveling wave structures, Technical Report, 1986, https://accelconf .web.cern.ch/186/papers/tu2-4.pdf.

[14] C. G. Montgomery, R. H. Dicke, and E. M. Purcell, Principles of microwave circuits (Iet, 1987), ISBN: 9780863411007

[15] S. G. Tantawi, R. D. Ruth, A. E. Vlieks, and Max Zolotorev, Active high-power RF pulse compression using optically switched resonant delay lines, IEEE Trans. Microwave Theory Tech. 45, 1486 (1997).

[16] L. Laurent, S. Tantawi, V. Dolgashev, C. Nantista, Y. Higashi, M. Aicheler, S. Heikkinen, and W. Wuensch, Experimental study of rf pulsed heating, Phys. Rev. ST Accel. Beams 14, 041001 (2011).

[17] S. G. Tantawi, V. Dolgashev, Y. Higashi, and B. Spataro, Research and development for ultra-high gradient accelerator structures, AIP Conf. Proc. 1299, 29 (2010).

[18] D. P. Pritzkau, G. B. Bowden, A. Menegat, and R. H. Siemann, Possible high power limitations from RF pulsed heating, AIP Conf. Proc. 474, 387 (1999).

[19] D. P. Pritzkau, rf pulsed heating, Technical Report, 2001, https://www.slac.stanford.edu/pubs/slacreports/reports13/ slac-r-577.pdf.

[20] Z. Li et al., RDDS cell design and optimization for the linear collider linacs, in Proceedings of the 18th Particle Accelerator Conference, New York, 1999 (IEEE, New York, 1999), Vol. 5.

[21] M. Nasr, P. Welander, Z. Li, and S. Tantawi, in Proceedings of the 10th International Particle Accelerator Conference (IPAC'19), Melbourne, Australia, 2019, http://accelconf .web.cern.ch/ipac2019/papers/weprb090.pdf.

[22] M. Nasr and S. Tantawi, in Proceedings of the 9th International Particle Accelerator Conference (IPAC'18),
Vancouver, BC, Canada, 2018 (JACOW Publishing, Geneva, Switzerland, 2018), pp. 4395-4397, http:// accelconf.web.cern.ch/ipac2018/papers/thpmk049.pdf.

[23] T. Argyropoulos et al., Phys. Rev. Accel. Beams 21, 061001 (2018).

[24] C. Adolphsen et al., in Proceedings of the 22nd Particle Accelerator Conference, PAC-2007, Albuquerque, NM (IEEE, New York, 2007).

[25] P. P. J. Willoughby, Elastically averaged precision alignment, Ph.D. thesis, Massachusetts Institute of Technology, 2005.

[26] P. Borchard, S. Appert, and J. Hoh, Fabrication of splitsection X-band structure using elastic averaging, J. Phys. Conf. Ser. 1067, 082002 (2018).

[27] C. W. Steele, A nonresonant perturbation theory, IEEE Trans. Microwave Theory Tech. 14, 70 (1966).

[28] J. Wang, Accelerator structure development for NLC/GLC, Technical Report, Stanford Linear Accelerator Center, Menlo Park, CA, 2004, https:/www.osti.gov/biblio/ 826798.

[29] S. G. Tantawi, C. D. Nantista, V. A. Dolgashev, C. Pearson, J. Nelson, K. Jobe, J. Chan, K. Fant, J. Frisch, and D. Atkinson, High-power multimode $\mathbf{X}$-band rf pulse compression system for future linear colliders, Phys. Rev. ST Accel. Beams 8, 042002 (2005).

[30] M. Nasr and S. Tantawi, in Proceedings of the 8th International Particle Accelerator Conference (IPAC'17), Copenhagen, Denmark, 2017 (JACOW, Geneva, Switzerland, 2017), pp. 1634-1636, http://accelconf.web .cern.ch/ipac2017/papers/tupab132.pdf.

[31] M. Nasr and S. Tantawi, in Proceedings of the 9th International Particle Accelerator Conference (IPAC'18), Vancouver, BC, Canada, 2018, pp. 4391-4394, http:// accelconf.web.cern.ch/ipac2018/papers/thpmk048.pdf.

[32] P. Welander, Z. Li, M. Nasr, and S. Tantawi, in Proceedings of the 9th International Particle Accelerator Conference (IPAC'18), Vancouver, BC, Canada, 2018, pp. 38353837, http://accelconf.web.cern.ch/ipac2018/papers/thpa 1080.pdf.

[33] K. Johnsen, Heavy beam loading in linear electron accelerators, Proc. Phys. Soc. London Sect. B 64, 1062 (1951).

[34] G. Saxon, Theory of Electron Beam Loading in Linear Accelerators, Proc. Phys. Soc. London Sect. B 67, 705 (1954).

[35] R. B. Neal, J. Appl. Phys. 29, 1019 (1958); W. W. Hansen Laboratories, M. L. Report No. 379, Stanford, 1957. 